\title{
Compact Beta Particle/Positron Imager for Plant Biology
}

\author{
A.G. Weisenberger, A. Stolin, B. Kross, S.J. Lee, S. Majewski, J. McKisson, J.E. McKisson, W. Xi, C. Zorn, C. \\ R. Howell, A.S Crowell, C.D. Reid and M.F. Smith
}

\begin{abstract}
The ${ }^{11} \mathrm{CO}_{2}$ tracer is used to facilitate plant biology research towards optimization of plant productivity, biofuel development and carbon sequestration in biomass. Positron emission tomography (PET) imaging has been used to study carbon transport in live plants using ${ }^{11} \mathrm{CO}_{2}$. Many plants typically have very thin leaves resulting in little medium for the emitted positrons to undergo an annihilation event. For the emitted positron from ${ }^{11} \mathrm{C}$ decay approximately $1 \mathrm{~mm}$ of water equivalent material is needed for positron annihilation. Thus most of the positrons do not annihilate inside the leaf, resulting in limited sensitivity for PET imaging. To address this problem we have developed a compact beta-positive beta-minus particle (BPBM) imager for ${ }^{11} \mathrm{CO}_{2}$ leaf imaging. The detector is based on a Hamamatsu $\mathrm{H8500}$ position sensitive photomultiplier tube optically coupled via optical grease and a $3 \mathrm{~mm}$ thick glass plate to a $0.5 \mathrm{~mm}$ thick Eljin EJ-212 plastic scintillator. The detector is equipped with a flexible arm to allow its placement and orientation on the leaf of the plant of interest to maintain the original leaf orientation. We are planning to utilize the imaging device at the Duke University Phytotron to investigate dynamic carbon transport differences between invasive and native vines.
\end{abstract}

\section{INTRODUCTION}

A $\mathrm{s}$ the first physiological studies using short-lived radioisotopes were in plant physiology before medical applications, a renewed interest in radioisotopes for plant ecophysiology can benefit from improved imaging techniques. Radionuclide imaging has for over half a century been providing insight into biological processes pertinent to medical health care. Recently specialized radionuclide imaging systems

Manuscript received November 21, 2010. The Jefferson Science Associates (JSA) operates the Thomas Jefferson National Accelerator Facility for the United States Department of Energy under contract DE-AC05-06OR23177. Support for this research came from, the DOE Office of Biological and Environmental Research, the DOE Office of Nuclear Physics grant DE-FG0297ER41033 and National Science Foundation grant nos. IBN-9985877 and DBI-0649924.

A.G. Weisenberger, B. Kross, S.J. Lee, J. McKisson, J. McKisson C. Zorn are with Jefferson Lab, Newport News, VA, USA, (telephone: 757-269-7090, e-mail: drew@jlab.org).

C. Howell, A. Crowell and C.D. Reid are with Duke University \& TUNL, Durham, NC, USA, (telephone: 919-660-2632, e-mail: howell@tunl.duke.edu).

M.F. Smith is with the University of Maryland, Baltimore, MD, USA, (telephone: 410-328-1320, e-mail: msmith7@umm.edu).

A. Stolin and S. Majewski are with West Virginia University, Morgantown, WV, USA, (telephone: 304-293-6929, e-mail: astolin@hsc.wvu.edu). have been developed to accomplish specific radioisotope imaging tasks for nuclear medicine and biomedical research.

We are collaborating on detector development to aid plant biology research involving plant ecophysiological and microbial research and to test and use the technology in current and proposed plant research on determining the direct impact of $\mathrm{CO}_{2}$ on biomass and carbon sequestration in species from forest ecosystems.

The ${ }^{11} \mathrm{CO}_{2}$ tracer is favored for use in plant biology research. Positron emission tomography (PET) imaging has been used to study carbon transport in live plants using ${ }^{11} \mathrm{CO}_{2}$. Radiotracer studies utilizing a small two-headed PET system for plant research are underway at the Phytotron at Duke University and use the TUNL tandem Van de Graaff accelerator [1, 2]. Instrumentation originally developed for small animal PET has been modified for imaging ${ }^{11} \mathrm{C}$ in plant research as currently undertaken at Institute Phytosphere, Forschungszentrum Jülich in Germany [3]. Another twoheaded planar PET plant imaging system for ${ }^{11} \mathrm{C}$ has also recently been described by a research group in Japan [4]. At Brookhaven National Laboratory researchers have used a clinical human PET scanner to track the distribution of ${ }^{11} \mathrm{C}$ labeled metabolites in plants in response to environmental changes [5]. From these examples it is clear there is a strong interest in using ${ }^{11} \mathrm{C}$ for plant biology research.

Many plants typically have very thin leaves resulting in little medium for the emitted positrons to undergo an annihilation event. For the emitted positron from ${ }^{11} \mathrm{C}$ approximately $1 \mathrm{~mm}$ of water equivalent material is needed for a positron annihilation as the end point energy of the ${ }^{11} \mathrm{C}$ positron is $0.96 \mathrm{MeV}$. Thus most of the positrons do not annihilate inside the leaf of the plant, resulting in limited sensitivity of the PET images. With these PET imaging limitations in mind we have developed a compact beta-plus beta-minus particle (BPBM) imager for ${ }^{11} \mathrm{CO}_{2}$ leaf imaging. This is an expansion of our earlier effort using plastic scintillator coupled to a $110 \mathrm{~cm}$ diameter Hamamatsu R3292 PSPMT. In that work we used a rose leaf whose stem was placed for 90 minutes in a solution containing ${ }^{18} \mathrm{~F}$ radioisotope [6]. Please see Fig. 1. The ${ }^{18} \mathrm{~F}$ is often used as a surrogate for water to study short-term dynamics of water transport [7]. 


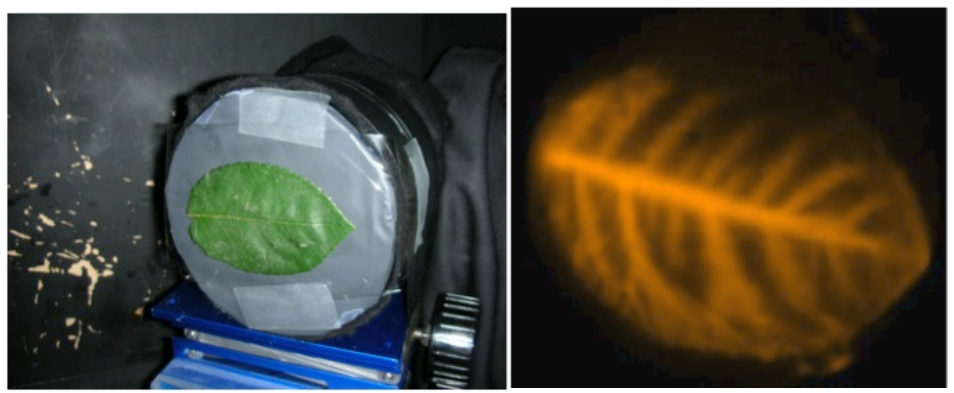

Figure 1. (Left) Rose leaf permeated with F-18 imaged with Positron Detector. (Right) Positron image of the rose leaf. Activity at the beginning of the 60 minute data acauisition was $150 \mathrm{nCi}$.

\section{METHODS}

The BPBM detector is based on a single Hamamatsu H8500 multi-anode photomultiplier tube (PSPMT) optically coupled via optical grease and a $3.18 \mathrm{~mm}$ thick glass plate to a $0.5 \mathrm{~mm}$ thick Eljin EJ-212 plastic scintillator [8]. Rexon RX688 optical grease was used on all optical surfaces [9]. The exposed surface of the plastic scintillator was encased in diffuse white Millipore paper. Photographs of the detector are shown in Fig. 2.

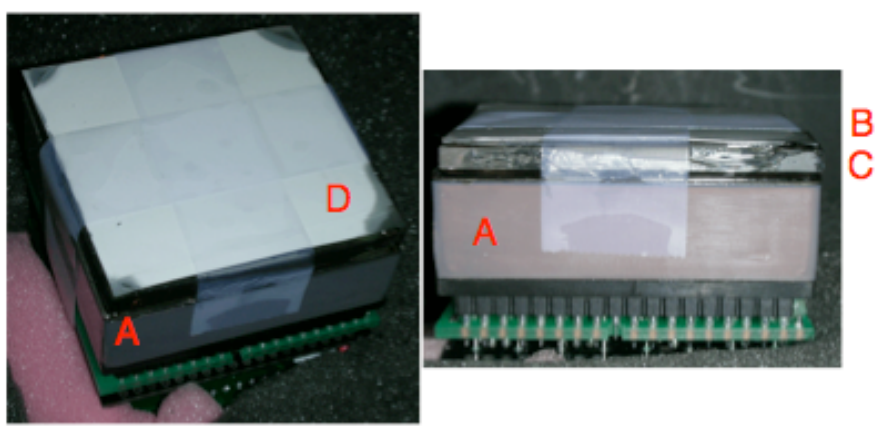

Fig. 2. Top and side view photographs of the detector. The Hamamatsu 8500 PSPMT (A) is optically coupled via a $3.18 \mathrm{~mm}$ glass spreader (C) to the $0.5 \mathrm{~mm}$ EJ-212 plastic scintillator (B). White Millipore paper (D) covers the top optical surface of the scintillator.

We employ a four-channel readout for the detector head [10]. The readout and image formation is accomplished using a Jefferson Lab developed FPGA based ADC interfaced to the PC via USB2 and a Java based data acquisition system [11, 12]. Final assembly and phantom testing of the device were accomplished at West Virginia University. An arrow shaped phantom was constructed using $2 \mathrm{~mm}$ diameter molecular sieve beads placed on a $5 \mathrm{~mm}$ grid. The beads were soaked with fluorine-18 solution. The phantom was sealed with a Saran Wrap type thin plastic wrap. Test images were obtained by placing the detector in contact with the plastic wrap covered beads.

\section{RESULTS}

The plot shown in Fig. 3 is a profile through four beads in the row of beads (right to left) placed at $45 \mathrm{deg}(7.1 \mathrm{~mm}$ spacing). The estimated resolution (including $2 \mathrm{~mm}$ bead size) is $\sim 3 \mathrm{~mm}$ FWHM.
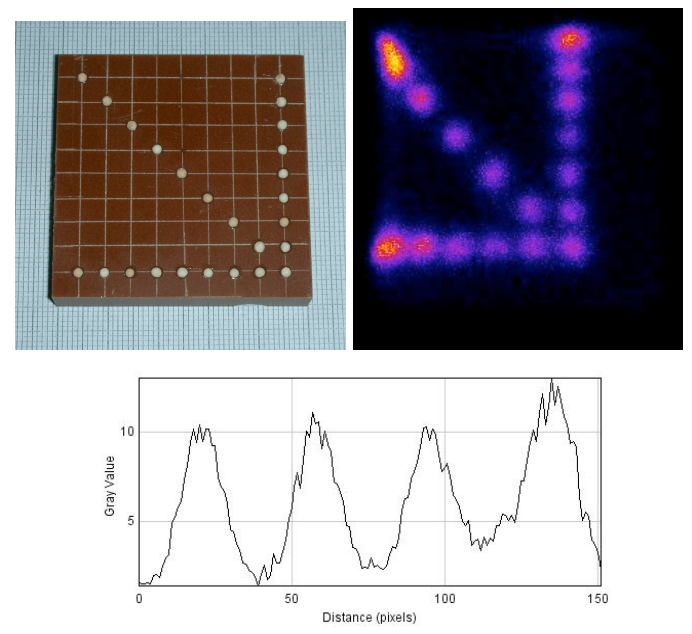

Fig. 3. Arrow phantom (top-left) and image (top-right) made with $2 \mathrm{~mm}$ diameter molecular sieve beads placed on a $5 \mathrm{~mm}$ grid. Beads were soaked with F18 solution and the image was obtained through a Saran wrap plastic covering. The plot (bottom) is a profile through four beads in the row of beads placed at $45 \mathrm{deg}$ ( $7.1 \mathrm{~mm}$ spacing). The estimated resolution (including $2 \mathrm{~mm}$ bead size) is $\sim 3 \mathrm{~mm}$ FWHM.

The detection efficiency was measured to be $\sim 14.5 \%$. The PSPMT based detector is housed in a $4.75 \mathrm{~mm}$ thick aluminum housing. The PSPMT frame and window frame is made from black Delrin from DuPont. A $0.05 \mathrm{~mm}$ thick Tedlar film from DuPont is used as a light tight entrance window. The detector is attached to a flexible arm to allow its placement on the area of the plant of interest to the researcher. This compact system allows for easy placement in environmental growth chambers. Please see Fig.4.

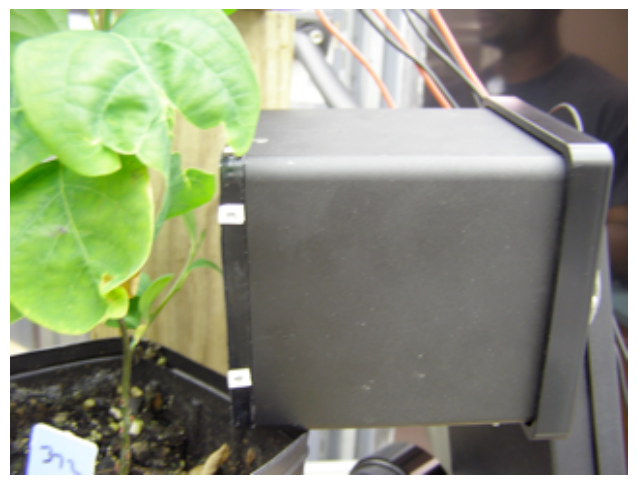

Fig. 4. Photograph of BPBM imager next to spicebush.

The BPBM detector was used to monitor the uptake of ${ }^{11} \mathrm{CO}_{2}$ by the spicebush shrub (Lindera benzoin). The spicebush is a native shrub used in studies to compare invasive species by measuring ${ }^{11} \mathrm{CO}_{2}$ to carbohydrate utilization. In tests at the Duke University Phytotron we introduced ${ }^{11} \mathrm{CO}_{2}$ in to the plant through one of its leaves through a cuvette, which was sealed around half of one leaf (see Fig. 5.) 


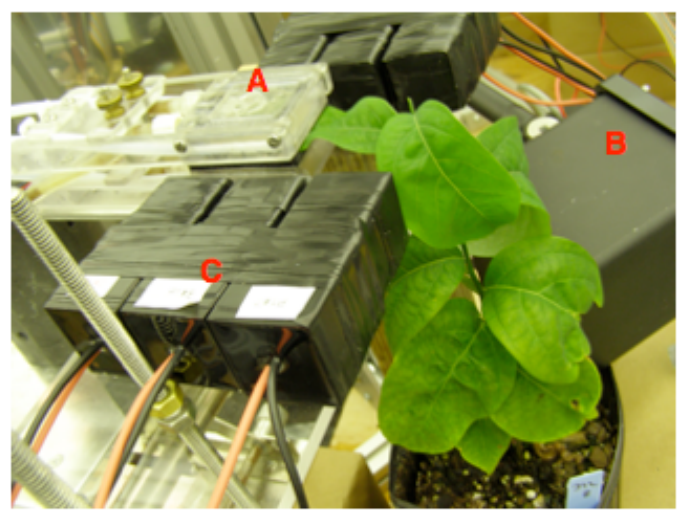

Fig. 5. Photograph of spicebush with one of its leaves contained in a clear plastic cuvette (A) which acts as the labeling chamber. The BPBM imager (B) is placed near the main stem of the bush. Coincidence detectors $(\mathrm{C})$ are used to monitor the amount of ${ }^{11} \mathrm{C}$ in the cuvette.

In this particular study no signal was seen in the stem. If the ${ }^{11} \mathrm{CO}_{2}$ was absorbed by the leaf it should have been through photosynthesis been incorporated into carbohydrates and transported through the leaf to the stem. We removed the leaf from the cuvette and placed in near contact with the active area of the BPBM detector. The resulting image (see Fig. 6) provided an indication that perhaps the cuvette was sealed too tightly around the leave preventing the sugars from transporting through the leaf.

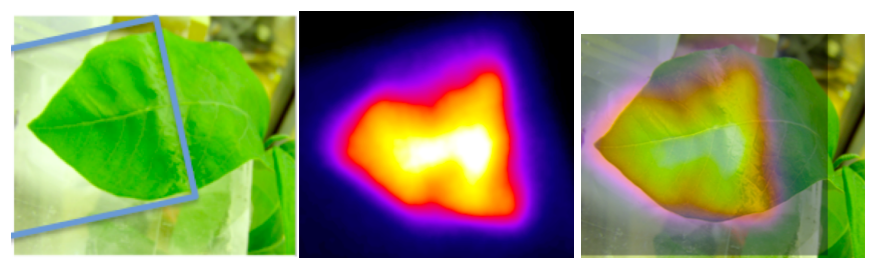

Fig. 6. Photograph of carbon dioxide induction leaf and image of uptake of ${ }^{11} \mathrm{CO}_{2}$. It appears the cuvette was sealed too tightly. The blue rectangle on the left photograph indicates the location of the cuvette.

\section{Discussion}

Based on the experience with studies described above we further modified the BPBM to make it more suitable for plant studies. The enclosure was made flatter and with less dead space at the front edge (see Fig. 7).

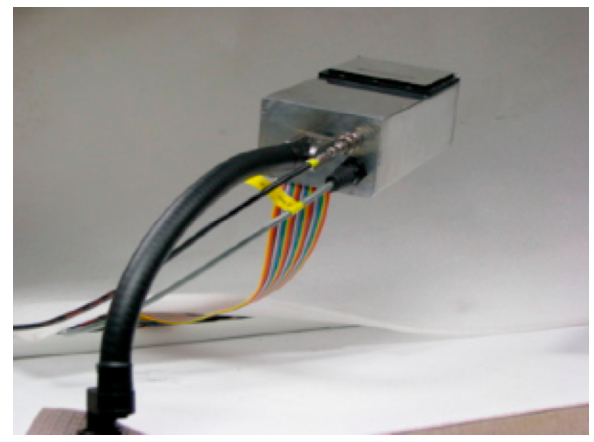

Fig. 6. Photograph of reconfigured BPPM detector. The detector is smaller in size and on a flexible arm for easier placement.
Additionally the surface of the enclosure was made reflective to reduce its interference with light to the plant thus impacting photosynthesis when positioned near the leaves of a plant. Additional studies were made with the modified detector and are being analyzed and will be reported on in a future publication.

\section{ACKNOWLEDGMENT}

We thank the staff at the TUNL tandem Van de Graaff accelerator laboratory and the Duke University Phytotron for their assistance.

\section{REFERENCES}

[1] Kiser MR, Reid CD, Crowell AS, Phillips RP, and Howell CR, (2008). "Exploring the Transport of Plant Metabolites Using Positron Emitting Radiotracers," HFSP J., vol. 2, 189-204, 2008.

[2] Weisenberger A.G., Kross B., Majewski S., McKisson J., Stolin A., Howell C.R., Crowell A.S., Reid C.D., Majewski S and Smith M.F., "Positron Emission Tomography Detector Development for Plant Biology," Conference Record of the 2009 IEEE Nuclear Science Symposium and Medical Imaging Conference, Orlando, J01-3, pp. 2323-2328, 2009.

[3] M. Streun, S. Beer (Weber), T. Hombach, S. Jahnke, M. Khodaverdi, H. Larue, S. Minwuyelet, C. Parl, G. Roeb, U. Schurr and K. Ziemons. "PlanTIS: A Positron Emission Tomograph for Imaging 11C Transport in Plants," IEEE Nuclear Science Symposium Conference Record, vol. 6: 4110-4112, 2007.

[4] N Kawachi, K Sakamoto, S Ishii, S Fujimaki, N Suzui, "Kinetic Analysis of Carbon-11-Labeled Carbon Dioxide for Studying Photosynthesis in a Leaf Using Positron Emitting Tracer Imaging System," IEEE Transactions on Nuclear Science, vol. 53, no. 5, pp. 2991-2997 October 2006.

[5] Jeremy Pritchard, A. Deri Tomos, John F. Farrar, Peter E. H. Minchin, Nick Gould, Matthew J. Paul, Elspeth A. MacRae, Richard A. Ferrieri, Dennis W. Gray and Michael R. Thorpe, "Turgor, solute import and growth in maize roots treated with galactose," Functional Plant Biology 31(11) 1095-1103, 18 November 2004.

[6] Stolin A., Weisenberger A.G., McKisson J, and Majewski, S., "Feasibility Study of Using Detection of Direct Positrons in Plant Imaging Research," Conference Record of the 2009 IEEE Nuclear Science Symposium and Medical Imaging Conference, Orlando, J01-7, pp. 2338-2341, 2009. 
[7] Nakanishi, T, et al. (2001b). "Comparison of 15Olabeled and 18F-labeled water uptake in a soybean plant by PETIS (Positron Emitting Tracer Imaging System)." Radioisotopes 50, 265-269.

[8] Eljen Technology, 2010 E. Broadway, Sweetwater Texas

[9] Rexon Components, Inc. 24500 High Point Road, Beachwood, $\mathrm{OH}$.

[10] V Popov, S Majewski, AG Weisenberger, Readout electronics for multianode photomultiplier tubes with pad matrix anode layout 2003 IEEE Nuclear Science Symposium Conference Record, 2004;3(3):21562159.

[11] J. Proffitt, W. Hammond, S. Majewski, V. Popov, R.R. Raylman, A.G. Weisenberger, R. Wojcik, R, "A flexible high-rate USB2 data acquisition system for PET and SPECT imaging," IEEE Nuclear Science Symposium Conference Record, vol. 5, pp: 2971 - 2975, Oct. 2005.

[12] J.E. McKisson, W. Hammond, J. Proffitt, and A.G. Weisenberger, "A Java Distributed Acquisition System for PET and SPECT Imaging", Conference Record, 2007 IEEE Nuclear Science Symposium and Medical Imaging Conference, Honolulu, October 27 - November 3, 2007. 